\title{
Journal Watch: Dr Tu looks back at the most important research articles released in 2018 relating to models and biomarkers of hepatocellular carcinoma
}

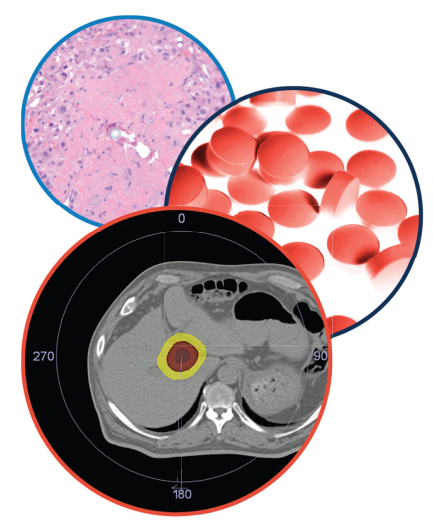

Hepatic Oncology

\author{
Thomas Tu*,1 \\ ${ }^{1}$ Department of Infectious Diseases, Molecular Virology, Heidelberg University Clinic, Heidelberg, Baden-Württemberg, Germany \\ *Author for correspondence: thomas.tu@med.uni-heidelberg.de
}

First draft submitted: 24 January 2018; Accepted for publication: 24 January 2018; Published online: 12 February 2019

Keywords: CRISPR • ctDNA • hepatocellular carcinoma • organoid • tumor suppressor genes

Wang G, Chow RD, Ye L et al. Mapping a functional cancer genome atlas of tumor suppressors in mouse liver using AAV-CRISPR-mediated direct in vivo screening. Sci. Adv. 4(2), eaao5508 (2018).

Multiple putative hepatocellular carcinoma (HCC) driver mutations have been identified using next-generation sequencing, but the majority have not been confirmed in in vivo settings or in combination with each other. Wang et al. describe a high-throughput solution by transducing transgenic mice containing a Cre-dependent Cas9 with hepatocyte-specific adeno-associated virus vectors that encode for Cre recombinase, EGFP and a sgRNA from a library, targeting HCC candidate genes ( 49 genes drawn from online databases). Indels at target loci were then confirmed and quantified using multiplexed molecular inversion probe sequencing.

Within 3 months of transduction, mice developed multifocal GFP-positive HCC, in which significant enrichment of Trp53, Setd2, Cic and Pik3R1 mutations were observed. Several pairs of mutations were significantly enriched, including Cdkn2a and Pten, B2m and Kansl1, and Arid2 and Cdkn2a. This exciting novel HCC model has the potential to confirm thousands of gene candidates identified by sequencing projects.

Nuciforo S, Fofana I, Matter MS, et al. Organoid models of human liver cancers derived from tumor needle biopsies. Cell Rep. 24(5), 1363-1376 (2018)

Hepatoma cell lines do not recapitulate many features of liver cancers, including histological morphology and subclonal heterogeneity. Nuciforo et al. describe a workflow to produce tumor organoids from primary tumor tissues (HCC and cholangiocarcinomas). The authors characterize histological, molecular and genomic features of the primary liver tumors, liver organoids and xenograft organoid tumors established in immunodeficient mice.

Tumors of multiple etiologies supported organoid establishment and culturing for up to 32 weeks. Coverage of different tumor genetic subtypes was observed. Histology and cancer markers expression of the primary tumor were both maintained in respective organoids and xenografts, suggesting these factors are independent of the liver microenvironment. Primary tumor subclonal mutations were observed at similar frequencies in the organoids, showing that a range of cells (and not a select few clones) establishes the organoids. This method opens up broad possibilities to observe more physiologically accurate processes in tumor biology.

Jiang $\mathrm{P}$, Sun $\mathrm{K}$, Tong $\mathrm{YK}$, et al. Preferred end coordinates and somatic variants as signatures of circulating tumor DNA associated with hepatocellular carcinoma. Proc. Natl Acad. Sci. USA.

115(46), E10925-E10933 (2018)

Given the genetic heterogeneity in HCCs, DNA mutations are difficult to use as biomarkers. Jiang et al. explore an alternate approach by characterizing DNA fragments in the circulation of HCC patients and healthy controls using PCR-free deep sequencing. The authors found that the size of circulating DNA fragments displays a nonrandom distribution and showed preferred sites of fragmentation. Interestingly, in HCC patients, both the fragment size

Future Medicine 
distribution and the sites of preferred fragmentation of the circulating DNA significantly change. Using a training set of a liver cancer patient, the authors define a 'cancer-associated' set of preferred fragment ends and then differentiated (with an area under the curve value of 0.88) HCC patients $(\mathrm{n}=90)$ from healthy, liver disease and liver cirrhosis controls $(n=32,67,36)$ by their circulating DNA fragments. This approach could present a novel way to detect HCC, despite its many genetic manifestations.

Financial \& competing interests disclosure

T Tu is supported by the German Center for Infection Research (DZIF), TTU Hepatitis Projects 5.807 and 5.704, and the Deutsche Forschungsgemeinschaft (DFG) TRR179 (TP 15), and the Australian Center for HIV and Hepatitis Virology Research. The author has no other relevant affiliations or financial involvement with any organization or entity with a financial interest in or financial conflict with the subject matter or materials discussed in the manuscript apart from those disclosed.

No writing assistance was utilized in the production of this manuscript.

Open access

This work is licensed under the Attribution-NonCommercial-NoDerivatives 4.0 Unported License. To view a copy of this license, visit http://creativecommons.org/licenses/by-nc-nd/4.0/ 